\title{
ORMDL3 may participate in the pathogenesis of bronchial epithelial-mesenchymal transition in asthmatic mice with airway remodeling
}

\author{
QI CHENG and YUNXIAO SHANG
}

Pediatric Pulmonology Department, Shengjing Hospital of China Medical University, Shenyang, Liaoning 110004, P.R. China

Received June 20, 2017; Accepted October 24, 2017

DOI: $10.3892 / \mathrm{mmr} .2017 .7972$

\begin{abstract}
Asthma is a common chronic respiratory disease in children that is caused by a complex interaction between genetic and environmental factors. Orosomucoid-like 3 (ORMDL3) is a candidate gene that has been strongly associated with asthma; however, the underlying mechanisms are unknown. ORMDL3 regulates the expression of metalloproteinases and transforming growth factor- $\beta$, and ORMDL 3 transgenic mice exhibit increased airway remodeling. Therefore, ORMDL3 may be associated with airway remodeling. The present study attempted to examine the associations between ORMDL3 and the severity of airway remodeling in asthmatic mice, and also to determine whether ORMDL3 induces epithelial-mesenchymal transition (EMT) in the bronchial epithelium. For this purpose, BALB/c mice were randomly assigned to control and asthma groups. Lung tissues were collected on days 3, 7 and 14 of the ovalbumin (OVA) challenge. Airway remodeling in asthmatic mice was then observed by hematoxylin and eosin, and Masson staining. Morphological changes in the bronchial epithelium were assessed by transmission electron microscopy. The EMT-associated indicators E-cadherin (E-cad), fibroblast-specific protein 1 (FSP1) and Vimentin (VIM) were assessed by western blotting and reverse transcription-quantitative polymerase chain reaction (RT-qPCR) at different time points of airway remodeling in asthmatic mice to detect the trend in EMT. Then, the localization of ORMDL3 was observed by immunohistochemistry, and its protein and mRNA expression was examined by western blotting and RT-qPCR, respectively. Furthermore, the bronchial epithelial cell line 16HBE14o-was transfected with an ORMDL3-expressing plasmid, and the differences in E-cad, FSP-1 and VIM expression were detected by immunofluorescence, western
\end{abstract}

Correspondence to: Professor Yunxiao Shang, Pediatric Pulmonology Department, Shengjing Hospital of China Medical University, 36 Sanhao Street, Heping, Shenyang, Liaoning 110004, P.R. China

E-mail: yunxiaoshang0523@163.com

Key words: orosomucoid-like 3, asthma, airway remodeling, epithelial-mesenchymal transition, bronchial epithelium blotting and RT-qPCR; the cell invasive ability was assessed by microscopy. The results revealed that ORMDL3 expression in the bronchial epithelium was associated with airway remodeling and EMT progression in vivo. Transfection of ORMDL 3 into $16 \mathrm{HBE} 14 \mathrm{o}$-cells in vitro induced EMT. Taken together, these findings suggest that ORMDL3 may regulate EMT in the bronchial epithelium, thereby affecting airway remodeling in asthma.

\section{Introduction}

Asthma is one of the most common chronic respiratory diseases in the world. Clear evidence now suggests that asthma is a heterogeneous and genetically complex disease, with phenotypes conferred by both genetic susceptibility and environmental exposure. More than 100 genes have been identified in association with asthma (1), among which the orosomucoid-like 3 gene (ORMDL3) and the associated 17q21 locus have emerged in genome-wide association studies (GWAS) as likely contributors to the genetic susceptibility and underlying pathogenesis of asthma. This genetic association has been confirmed in ethnically diverse populations (2). Although the functions of ORMDL3 are incompletely understood, Balantic et al (3) have found that the genetic polymorphism rs4795405 in ORMDL3 is closely related not only to asthma but also to chronic obstructive pulmonary disease (COPD). Thus, ORMDL3 may be associated with airway remodeling in asthma.

Airway remodeling refers to pathological changes in severe and refractory asthma that include structural changes in the airway wall caused by chronic inflammation, resulting in persistent or progressive damage to lung function. The principal features of airway remodeling are airway epithelial damage, goblet cell hyperplasia and metaplasia, subepithelial fibrosis and increased smooth muscle mass. Numerous recent studies have shown that the airway epithelial cells are the first barrier in the airway and play an important role in airway remodeling $(4,5)$. Stimulation by allergens and environmental factors, as well as the release of inflammatory mediators and cytokines, may induce damage of the airway epithelial barrier, reduced adhesion among epithelial cells, down-regulation of epithelial genes, and up-regulation of interstitial cell genes (6). Epithelial cells may participate in airway remodeling through epithelial-mesenchymal transition (EMT) (5). 
Miller et al $(7,8)$ have observed that ORMDL3 is expressed in lung bronchial epithelial cells after allergen exposure and that it regulates the expression of metalloproteinases, TGF- $\beta$ and ADAM-8. ORMDL3 transgenic mice have increased levels of airway remodeling and exhibit characteristics similar to those of asthma, including hypertrophy of airway smooth muscles and increased epithelial fibers and lumen mucus $(7,8)$. Therefore, we propose that ORMDL3 may induce bronchial EMT under chronic inflammatory stimulation.

If ORMDL3 is closely related to human asthma, the expression of ORMDL3 in asthma, the relationship between ORMDL3 and the severity of airway remodeling in asthma, and the ability of ORMDL3 to induce EMT in the airway warrant further exploration. Humans and mice both express three ORMDL family members, and ORMDL3 has $96 \%$ homology between these two species, supporting the use of mice as an appropriate animal model to study the function of ORMDL3 in asthma (9). Thus, in this study, we observed the trends of ORMDL3 and the EMT-related indicators E-cadherin (E-cad), fibroblast-specific protein 1 (FSP1), and Vimentin (VIM) at different time points of airway remodeling in an asthmatic mouse model. Furthermore, an ORMDL3 plasmid was used to transfect the bronchial epithelial cell line $16 \mathrm{HBE}-14 \mathrm{o}-$, and the invasive ability of 16HBE $14 \mathrm{o}-\mathrm{cells}$ was assessed by microscopy. In addition, changes in the expression of E-cad, VIM, and FSP-1 were assessed to determine whether ORMDL3 induces EMT in bronchial epithelial cells.

\section{Materials and methods}

Asthmatic mouse model. A total of 56 specific-pathogen-free female BALB/c mice ( 8 weeks old) were purchased from the experimental animal research center of Shengjing Hospital of China Medical University (Shenyang, China). All experimental protocols involving animals were approved by the China Medical University Animal Care Committee and complied with the guidelines of the China Council on Animal Care. The animals were randomly assigned to the control group $(n=24)$, the model group $(n=24)$ and the treatment group $(n=8)$ at the beginning of the experiment. The mice in the model group were prepared according to previously described methods using a modified ovalbumin (OVA; O1641; Sigma-Aldrich, St. Louis, MO, USA) immunization protocol developed to induce allergic asthma in mice (10). The mice were initially intraperitoneally immunized with $20 \mu \mathrm{g}$ of OVA and $2 \mathrm{mg}$ of aluminum hydroxide gel (Sigma-Aldrich) in $0.5 \mathrm{ml}$ of PBS on days 0,7 , and 14 , as previously reported (11). Control mice were injected with saline only. From day 16 onward, the animals were treated every other day for 14 days as follows. The mice in the model group were placed in a

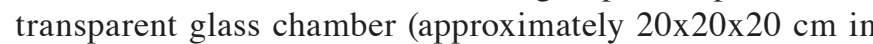
volume) connected to an ultrasonic nebulizer (model 100; Yadu, Shanghai, China) and subjected to repeated bronchial allergen challenge by inhalation of OVA (4\%) for $30 \mathrm{~min} /$ day. The mice in the control group were challenged with phosphate buffer saline (PBS). The mice in the dexamethasone treatment group were OVA sensitized and intraperitoneal injected with $0.2 \mathrm{ml}$ dexamethasone $(2 \mathrm{mg} / \mathrm{ml})$ before OVA challenged. On days 3,7 , and 14 of allergen challenge, 8 mice from control group and asthma group were anesthetized by intraperitoneal injection with 5\% chloral hydrate and sacrificed. While all of the treatment group were sacrificed on days 14 of challenged.

Whole lungs were collected aseptically by chest opening. The left lungs were fixed in paraformaldehyde (PFA) for subsequent immunohistochemical staining; the right upper lung lobes were used for real-time PCR analysis, and the right lower lung lobes were used for western blot analysis. All specimens were snap-frozen in liquid nitrogen and stored at $-80^{\circ} \mathrm{C}$ until use.

Lung histopathology. The left lungs were fixed in 4\% PFA for 18-24 h, embedded in paraffin and then routinely processed. Serial 5- $\mu \mathrm{m}$ tissue sections were stained with hematoxylin and eosin (H\&E) for general histological evaluation. Masson's trichrome staining was performed for the assessment of collagen particles and airway remodeling.

Morphometric analysis of the airway.H\&E-and Masson-stained tissue sections were examined by light microscopy at magnification, $x 200$, and morphometric measurements were obtained using Image-Pro Plus 6.0 software (Media Cybernetics, Inc., Rockville, MD, USA) to assess bronchiolar remodeling and collagen deposition. For H\&E-stained lung sections from each group, the complete cross-section of the bronchiole without cartilage was selected for analysis. The total bronchial wall area (Wat, $\mu \mathrm{m}^{2}$ ) and the diameter of the basal membrane perimeter (Pbm, $\mu \mathrm{m})$ were analyzed for each bronchiole. Wat/Pbm expresses the wall thickness of the bronchiole wall. Masson staining was used to assess the deposition of collagen. Two complete bronchioles were selected from each tissue section, and the percentage of the area of collagen deposition in the total stained area was calculated $(12,13)$.

Transmission electron microscopy. After dissection, lung specimens were fixed in $2.5 \%$ glutaraldehyde overnight at $4^{\circ} \mathrm{C}$. The tissues were washed in phosphate buffer ( $\mathrm{pH} 7.4$ ), postfixed in $1 \%$ osmium tetroxide in phosphate buffer, dehydrated in graded ethanol solutions, treated in propylene oxide and embedded in epoxy-resin embedding media. Thin transverse random sections (60 nm) were collected on single copper slot grids coated with parlodion, stained with uranyl acetate and lead citrate, and observed with a transmission electron microscope (H-7650; Hitachi Limited, Tokyo, Japan). Images were documented using Kodak SO163 EM film (Kodak, Rochester, NY, USA).

Immunohistochemistry. ORMDL3 and FSP-1 protein expression in the lung was detected by immunohistochemistry. Lung sections $(5 \mu \mathrm{m})$ were cut, blocked with peroxide and non-immune animal serum, and incubated sequentially with anti-ORMDL3 antibody (dilution, 1:200; LS-B 9583; LifeSpan BioSciences, Inc., Seattle, WA, USA) or FSP-1 antibody (dilution, 1:200; 16105-1-AP; Proteintech, Wuhan, China), biotin-labeled secondary antibody, and streptomycin anti-biotin peroxidase. Finally, the sections were stained with DAB, counterstained with hematoxylin, dehydrated, cleared in xylene, and fixed. Negative staining controls were generated by replacing the primary antibody with PBS. A laser scanning confocal microscope (MTC-600; Bio-Rad, Hercules, CA, USA) was used for image acquisition, and the deposition of brown particles in the cytoplasm indicated a positive result. 
Cell culture and transfection. Immortalized SV-40 virus-transformed 16HBE 140-human bronchial epithelial cells were purchased from the cell line resource of XiangYa School of Medicine. The cells were cultured in RPMI 1640 medium (SH30809.01B; HyClone; GE Healthcare, Logan, UT, USA) containing 12\% fetal bovine serum (FBS; SH30084.03; HyClone; GE Healthcare), penicillin (100 U/ml), and streptomycin $(100 \mu \mathrm{g} / \mathrm{ml})$ at $37^{\circ} \mathrm{C}$ in a humidified $5 \% \mathrm{CO}_{2}$ atmosphere. $16 \mathrm{HBE}-14 \mathrm{o}$ cells were efficiently transfected with either the GV230-ORMDL3-eGFP plasmid, which encodes the full-length ORMDL3 cDNA with a terminal GFP tag, or empty vector containing the GFP tag (eGFP-control) as a negative control (GeneChem, Shanghai, China) using Lipofectamine 2000 (11668019; Invitrogen, Carlsbad, CA, USA) according to the manufacturer's instructions to generate the GFP-ORMDL3 group and GFP-vector group, respectively.

Transwell analysis. To study the role of ORMDL3 in 16HBE 14o-migration, Transwell analysis was conducted after harvesting the transfected cells with trypsin and resuspending them $\left(1.0 \times 10^{6}\right.$ cells $\left./ \mathrm{ml}\right)$ in serum-free growth medium. The two groups of cells were added to the upper chamber. After incubation for $24 \mathrm{~h}$ at $37^{\circ} \mathrm{C}$, the membranes were removed, the cells on the upper side were scraped off, and the cells that migrated to the lower side of the membrane were fixed with $4 \%$ polyoxymethylene. The number of cells was counted in 5 random fields under magnification, $\mathrm{x} 20$, and the mean was calculated.

Immunocytochemistry. The two groups of 16HBE 140-cells were cultured on coverslips, and protein expression (E-cad, FSP-1) was detected by immunocytochemistry. After the cells reached confluency, they were fixed in $4 \%$ polyphosphate formaldehyde, blocked with peroxide and non-immune animal serum, and incubated sequentially with primary antibody (anti-E-cad; dilution, 1:50; ab11512, Abcam, Cambridge, UK; anti-FSP-1; dilution, 1:50; Santa Cruz, CA, USA) at $4^{\circ} \mathrm{C}$. Rat IgG (for E-cad) and Goat IgG (for FSP-1) were used as controls, and Cy3-conjugated donkey anti-goat IgG (dilution, 1:200; Proteintech) and goat anti-rat IgG (dilution, 1:200; Santa Cruz) were used as the secondary antibodies. The slides were washed, mounted in medium containing 4,6-diamidino-2-phenylindole (DAPI) and visualized by confocal microscopy (x400) at ambient temperature.

Western blot analysis. Total protein extracted from lung tissue and $16 \mathrm{HBE}-14 \mathrm{o}$ cells was quantified using a BCA protein assay kit. A $20-\mu l$ aliquot of protein was then loaded onto a $12 \%$ SDS-PAGE gel and transferred onto polyvinylidene difluoride membranes (Millipore, Billerica, MA, USA). The membranes were blocked with $5 \%$ skimmed milk for $1 \mathrm{~h}$ and then incubated with primary antibody (dilution, 1:800, ORMDL3; dilution, 1:5,000, E-cad; dilution, 1:1,000 FPS-1; dilution, 1:200 VIM; and dilution, 1:200, GAPDH) diluted in PBS overnight at $4^{\circ} \mathrm{C}$. After washing in Tris-buffered saline $+1 \%$ Tween-20 (TBST), the membranes were incubated for $2 \mathrm{~h}$ with horseradish peroxidase-conjugated secondary antibody (dilution, 1:500) and then imaged using enhanced chemiluminescence reagents. The density of the protein bands was analyzed using ImageJ software. GAPDH was used as an internal control.
Real-time PCR. Total RNA was isolated following the manufacturer's instructions using TRIzol reagent (Invitrogen, Carlsbad, CA, USA) and RNAiso ${ }^{\mathrm{TM}}$ Plus reagent (Takara, Dalian, China) and quantified using a spectrophotometer. Following quantification, $2 \mu \mathrm{g}$ of RNA was reverse-transcribed into cDNA, and real-time quantitative PCR assays were conducted using an ABI PRISM 7500 Real-time PCR System (Applied Biosystems, Foster City, CA, USA) and the SYBR PrimeScript $^{\mathrm{TM}}$ RT-PCR kit reagent (Takara, Dalian, China). The PCR conditions for NK-1R were 40 cycles of denaturation at $95^{\circ} \mathrm{C}$ for $5 \mathrm{sec}$ and annealing and extension at $60^{\circ} \mathrm{C}$ for $30 \mathrm{sec}$. Target mRNA levels were normalized to those of GAPDH. The oligonucleotide primers used are presented in Table I.

Statistical analysis. SPSS 17 software was used for statistical analysis. Inter-group comparisons were performed using the t-test, multiple group comparisons were conducted using one-way analysis of variance (ANOVA), and correlation analysis was performed using Pearson's test. All data are expressed as the mean $(\mathrm{X}) \pm$ standard deviation (SD). $\mathrm{P}<0.05$ was considered to indicate a statistically significant difference.

\section{Results}

Airway remodeling increases with the development of asthma. The development of airway remodeling was assessed in lung tissues stained with $\mathrm{H} \& \mathrm{E}$ and Masson's trichrome. We observed the level of peribronchial cellular infiltration and evaluated bronchial wall thickness and lung collagen content in all experimental mice. Representative sections of each group were stained with either H\&E (Fig. 1A-C) or Masson's trichrome (Fig. 1E-G). As shown in Fig. 1A, no inflammation, mucosal edema or epithelial lesions were observed in the control group. Mild inflammation, mucosal edema and epithelial lesions were observed in the asthma group at 3 days, whereas sensitization to OVA at 7 days and 14 days was associated with predominantly moderate to severe inflammation, mucosal edema and epithelial lesions that included interstitial infiltrates and large lymphoid aggregates (Fig. 1B). Bronchial wall thickness was quantitatively estimated based on $\mathrm{H} \& \mathrm{E}$ staining (Fig. 1D) and was observed to increase with the duration of OVA exposure $(\mathrm{P}<0.01)$.

As the duration of OVA exposure increased, the peribronchial trichrome-stained area and lung collagen levels were increased at 3, 7 and 14 days (Fig. 1F) of asthma group compared with those of the control group (Fig. 1E). The collagen content was quantitatively estimated (Fig. 1H) by Masson staining and was observed to increase with an increasing duration of OVA exposure $(\mathrm{P}<0.01)$. While in the treatment group, bronchial wall thickness was reduced and the collagen content reduced compared with the asthmatic model group; however, it was still higher than the control group, respectively (Fig. 1C, D, G and $\mathrm{H})(\mathrm{P}<0.01)$.

EMT is exacerbated with the development of asthma. Morphological changes in bronchiolar epithelial cells were assessed by transmission electron microscopy. The control group (Fig. 2A) exhibited normal bronchial epithelial cells without edema, neatly arranged cilia, no or rare cytoplasmic 
Table I. Primer sequences used for reverse transcription-quantitative polymerase chain reaction.

\begin{tabular}{|c|c|c|}
\hline Gene name & Species & Primer sequences $\left(5^{\prime}-3^{\prime}\right)$ \\
\hline GAPDH & Human & $\begin{array}{l}\text { F: GAGAAGGCTGGGGCTCATTT } \\
\text { R: AGTGATGGCATGGACTGTGG }\end{array}$ \\
\hline ORMDL3 & Human & $\begin{array}{l}\text { F: GAGCATCCCGTTTGTGAGTGTC } \\
\text { R: CCATCTGCTCCCAGTGGGTTA }\end{array}$ \\
\hline E-cad & Human & $\begin{array}{l}\text { F: GAGTGCCAACTGGACCATTCAGTA } \\
\text { R: AGTCACCCACCTCTAAGGCCATC }\end{array}$ \\
\hline VIM & Human & $\begin{array}{l}\text { F: GCAGGAGGCAGAAGAATGGT } \\
\text { R: ACGAAGGTGACGAGCCATTT }\end{array}$ \\
\hline FSP-1 & Human & $\begin{array}{l}\text { F: GGGTGACAAGTTCAAGCTCAACAA } \\
\text { R: ATCATGGCGATGCAGGACAG }\end{array}$ \\
\hline GAPDH & Mouse & $\begin{array}{l}\text { F: CTACCCCCAATGTGTCCGTC } \\
\text { R: GGGATAGGGCCTCTCTTGCT }\end{array}$ \\
\hline ORMDL3 & Mouse & $\begin{array}{l}\text { F: CCCTGTGGGTTTGAACTCCTG } \\
\text { R: GTCGAAGTGAACCCGCTTCTG }\end{array}$ \\
\hline E-cad & Mouse & $\begin{array}{l}\text { F: ATTGCAAGTTCCTGCCATCCTC } \\
\text { R: CACATTGTCCCGGGTATCATCA }\end{array}$ \\
\hline VIM & Mouse & $\begin{array}{l}\text { F: CCTATGTGACCCGGTCCTCG } \\
\text { R: AAGGTCAAGACGTGCCAGAG }\end{array}$ \\
\hline FSP-1 & Mouse & $\begin{array}{l}\text { F: GGAGGCCCTGGATGTAATTGTG } \\
\text { R: CAACTTCATTGTCCCTGTTGCTG }\end{array}$ \\
\hline
\end{tabular}

Human gene primers were used for reactions using 16HBE14o- cells and mouse gene primers were used for reactions involving BALB/c mice. F, forward primer; R, reverse primer; ORMDL3, Orosomucoid-like 3; E-cad, E-cadherin; VIM, Vimentin; FSP-1, fibroblast-specific protein 1.

vacuoles, regularly shaped nuclei, mitochondria, endoplasmic reticulum dilation, thin subepithelial basal lamina, few collagen fibers, and intercellular tight linking. At 3 days, the asthma group exhibited disordered cilia and a slightly expanded endoplasmic reticulum. At 7 days, in addition to disordered cilia, the asthma group exhibited obvious dilation of mitochondria and the endoplasmic reticulum, basal lamina thickening, and collagen deposition in clusters. At 14 days, the asthma group exhibited shedding of the ciliated epithelium, undetached ciliated epithelial cells, significantly increased bronchial epithelial cells, mitochondrial swelling, endoplasmic reticulum expansion, increased cytoplasmic vacuoles, globally increased secretory cells, intercellular tight junction gaps, and increased basal lamina collagen fibers (Fig. 2B). However, in the treatment group, the damage of organelles and ciliate epithelium was alleviated by asthma group (Fig. 2C).

E-cad, FSP1, and VIM were evaluated as EMT-related indicators. FSP-1 is a fibroblast marker that indicates EMT when expressed in epithelial cells. Immunohistochemistryrevealed that FSP-1 expression was nearly absent in bronchial epithelial cells in the control group. However, some FSP-1 was observed on the bronchial epithelium in the asthma group at 3 days, and obvious FSP-1 staining on the bronchial epithelium was observed in the asthma group at 7 days, with even greater staining at 14 days (Fig. 2E). While FSP-1 expression decrease in the treatment group.
Western blotting (Fig. 3A) revealed that OVA challenge resulted in decreased E-cad expression and increased VIM and FSP-1 expression in the asthma group compared with the control group, consistent with EMT progression.

Real-time PCR demonstrated that the mRNA expression of E-cad (Fig. 3C), FSP-1 (Fig. 3D), and VIM (Fig. 3E) did not differ significantly between the asthma group at 3 days and the control group $(\mathrm{P}>0.05)$. At 7 and 14 days, E-cad expression (Fig. 3C) was significantly lower and FSP-1 (Fig. 3D) and VIM expression (Fig. 3E) was greater in the asthma group than in the control group $(\mathrm{P}<0.01)$.

The treatment group signifcantly increased the mRNA and protein expression of E-cad, and decreased the mRNA and protein expression of VIM and FSP-1 compared with asthma group (Fig. 3B-E) $(\mathrm{P}<0.01)$.

ORMDL3 expression in the bronchial epithelium is associated with airway remodeling and EMT progression in vivo. We examined the location of the ORMDL3 protein by immunohistochemical staining of lung tissue from the control and asthma groups exposed to OVA over time. After OVA challenge, ORMDL3 was expressed in both the bronchial epithelium and lymphocytes around blood vessels in the asthma group (Fig. 4B), alleviated in treatment group (Fig. 4C), but was poorly expressed in the control group (Fig. 4A).

We also examined ORMDL3 protein and mRNA levels in lung tissue. Compared with the control group, ORMDL3 

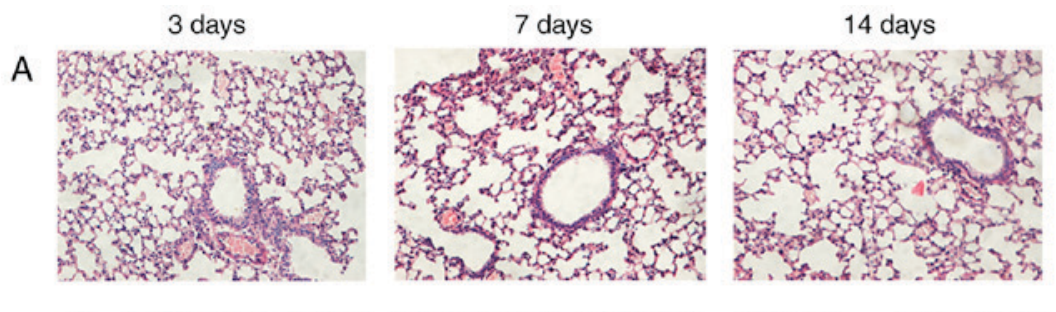

B
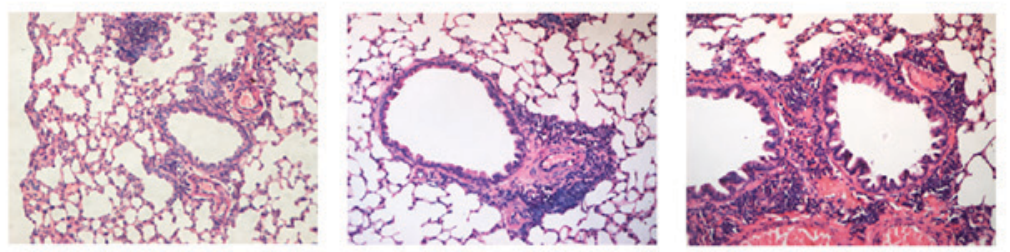

C
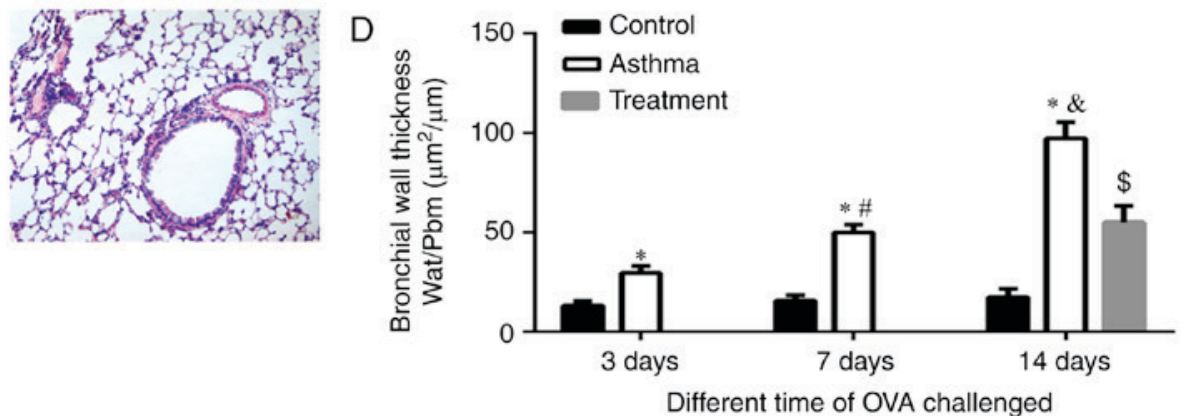

$E$
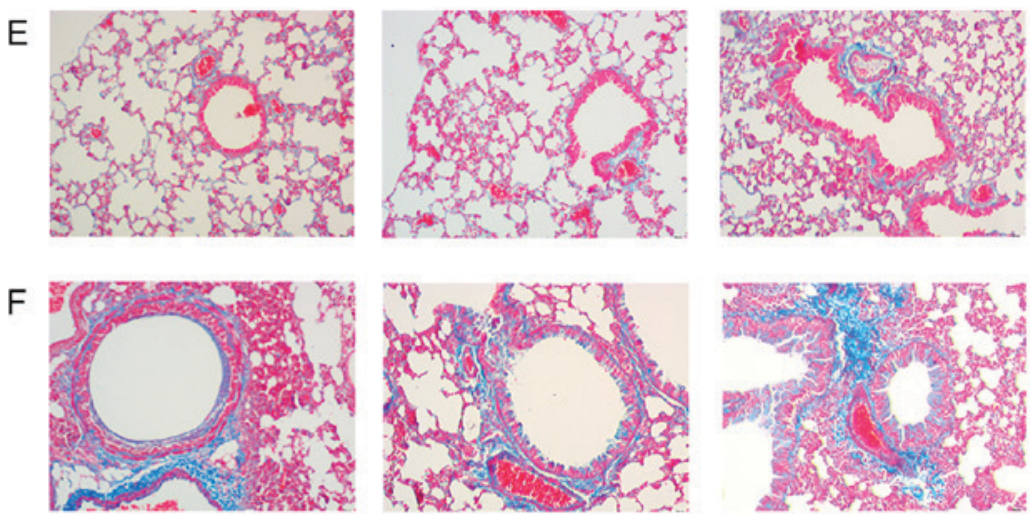

G

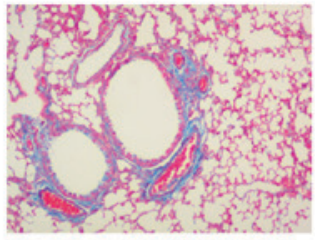

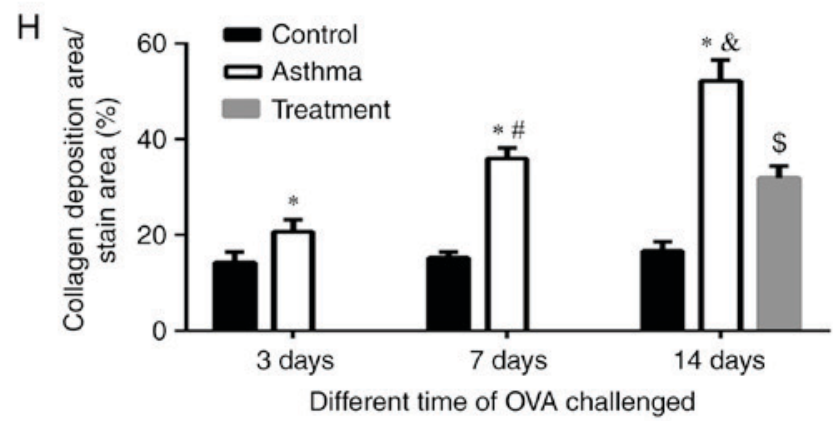

Figure 1. Airway remodeling increased with the development of asthma. H\&E staining of paraffin sections of lung tissues from BALB/c mice in the (A) control, (B) asthma and (C) treatment groups with different durations of exposure to OVA (magnification, $\mathrm{x} 200$ ). Masson staining of lung tissues from BALB/c mice in the (E) control, (F) asthma and (G) treatment groups with different durations of exposure to OVA (magnification, x200). Bronchial wall thickness (Wat/Pbm) was assessed by (D) H\&E staining, and the percentage of the area of collagen deposition was assessed by (H) Masson staining. Wat/Pbm, the ratio of the total bronchial wall area $\left(\right.$ Wat, $\left.\mu \mathrm{m}^{2}\right)$ to the diameter of the basal membrane perimeter $(\mathrm{Pbm})$, represents the thickness of the bronchial wall and was used to evaluate the severity of airway remodeling. The percentage of the area of collagen deposition was used to determine the severity of fibrosis in airway remodeling. " $\mathrm{P}<0.01$ vs. control; " $\mathrm{P}<0.01$ vs. asthma group at 3 days; ${ }^{\circledR} \mathrm{P}<0.01$ vs. asthma group at 7 days; ${ }^{\$} \mathrm{P}<0.01$ vs. treatment group. H\&E, hematoxylin and eosin; OVA, ovalbumin.

protein and mRNA expression was significantly increased in lung tissues from the asthma group upon OVA challenge (Fig. 4D, F; P<0.05). the treatment group signifcantly reduced the mRNA and protein expression of ORMDL3 (Fig. 4E, F)
$(\mathrm{P}<0.01)$. The expression of ORMDL3 mRNA was positively related to the expression of FSP-1 mRNA and VIM mRNA, bronchiolar wall thickness, and the percentage of collagen deposition but negatively related to the expression of E-cad 

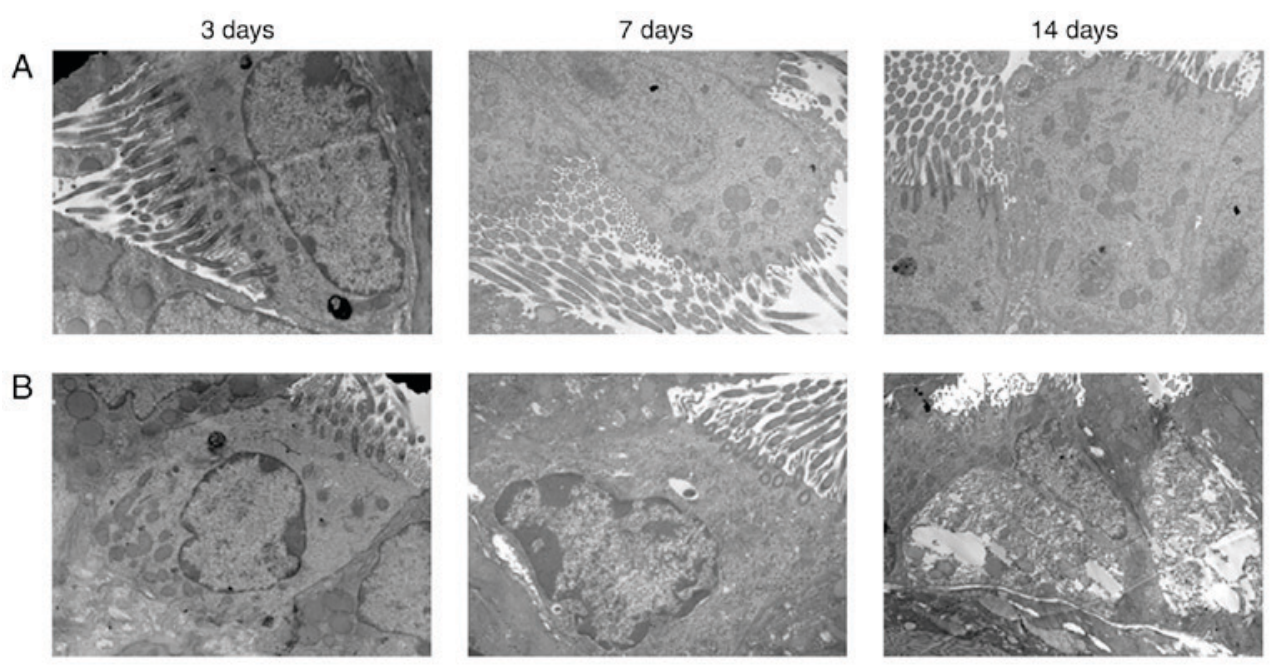

C
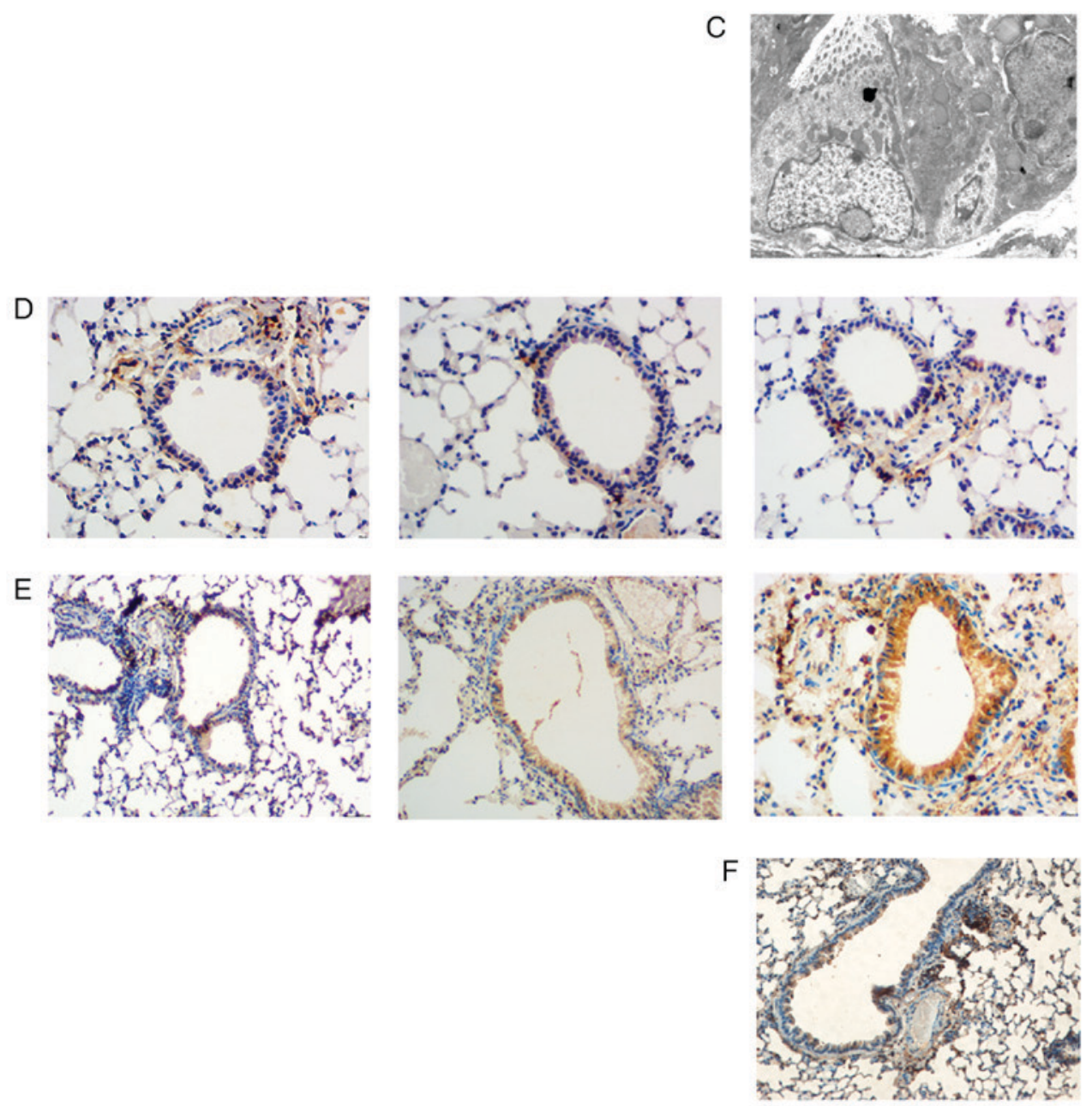

Figure 2. Morphological changes and FSP-1 localization of bronchial epithelium induced by the development of asthma. Electron microscopy of lung tissue focusing on bronchiolar cells in BALB/c mice from the (A) control group, (B) asthma groups and (C) treatment group with increasing durations of exposure to OVA (magnification, $\mathrm{x} 8,000$ ). FSP-1 immunohistochemistry of paraffin sections of lung tissue from BALB/c mice in the (D) control group, (E) asthma groups and (F) treatment group with increasing durations of exposure to OVA (magnification, x200). FSP-1, fibroblast-specific protein 1; OVA, ovalbumin.

mRNA (as indicated by Pearson's correlation coefficient, $\mathrm{P}<0.01$ ) (Table II).

Transfection of bronchial epithelial cell line 16HBE 140 with ORMDL3 induces EMT in vitro. Because our in vivo studies demonstrated that ORMDL3 expression increased in the airway epithelium along with bronchial EMT development with increasing duration of OVA allergen challenge, we examined whether ORMDL3 regulates the migration of airway epithelial cell-like mesenchymal cells and the expression of the EMT indicators FSP-1, VIM and E-cad in the human bronchial cell line 16HBE 140-. 16HBE14o- cells 

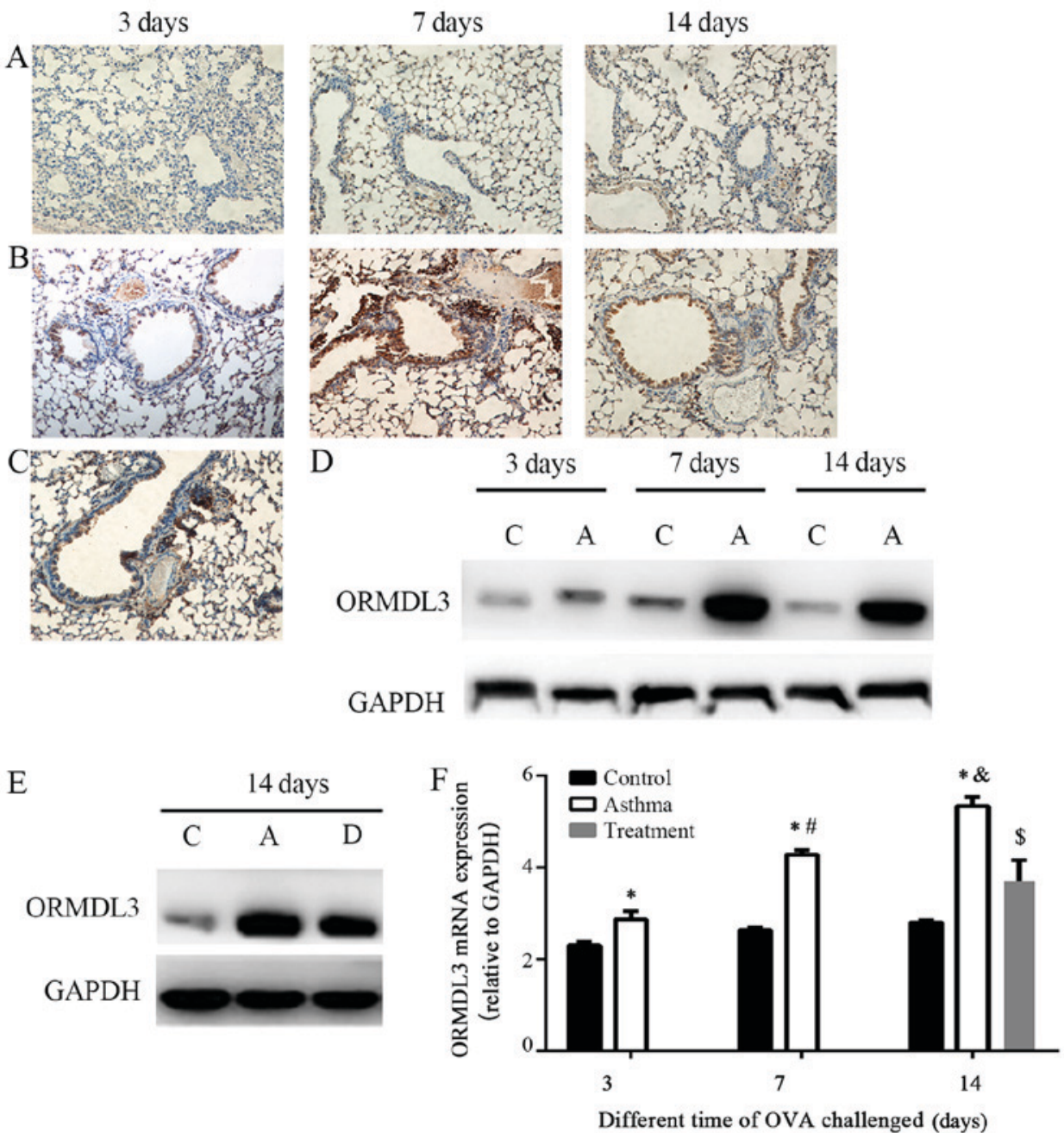

Figure 3. ORMDL3 expression in the bronchial epithelium is associated with airway remodeling and epithelial-mesenchymal transition development in vivo. ORMDL3 immunohistochemistry of paraffin sections of lung tissue from BALB/c mice in the (A) control and (B) asthma groups with increasing length of exposure to OVA, and (C) the treatment group with 14 days of exposure to OVA (magnification, x200). (D) Western blotting of ORMDL3 protein expression in the lung tissue of the control and asthma groups at different time points. (E) Western blotting of ORMDL3 protein expression in the lung tissue of the control, asthma and treatment groups with 14 days of exposure to OVA. (F) Reverse transcription-quantitative polymerase chain reaction of ORMDL3 mRNA expression in the lung tissue of the different groups. ${ }^{~} \mathrm{P}<0.01$ vs. control; ${ }^{\#} \mathrm{P}<0.01$ vs. asthma group at 3 days; ${ }^{\circ} \mathrm{P}<0.01$ vs. asthma group at 7 days; ${ }^{5} \mathrm{P}<0.01$ vs. treatment group. C, control group; A, asthma group; D, dexamethasone treatment group; ORMDL3, orosomucoid-like 3; OVA, ovalbumin.

were transfected with GFP-vector or GFP-ORMDL3, and the indicated protein levels were determined by western blot analysis (Fig. 5A). ORMDL3 expression was significantly lower in cells expressing GFP-vector than in those expressing GFP-ORMDL3. We also observed that cells expressing GFP-ORMDL3 had higher FSP-1 and VIM expression and lower E-cad expression than those expressing GFP-vector, as determined by western blotting (Fig. 5A) and real-time PCR (Fig. 5B). Immunofluorescence microscopy revealed that the transfection of ORMDL3 into 16HBE 14o-cells resulted in increased FSP-1 expression and decreased E-cad expression (Fig. 5C, D). The number of migrating cells was significantly increased among the cells transfected with GFP-ORMDL3 compared with those transfected with GFP-vector (Fig. 5E, F) (568.7 $\pm 5.7 / \mathrm{HP}$ vs. 403.0 $\pm 9.5 / \mathrm{HP})$.

\section{Discussion}

ORMDL3, a member of the ORM family, is located on chromosome 1q. The gene order of this chromosome is highly conserved from yeasts to vertebrates, and the ORMDL3 gene encodes a tetraspanin consisting of 153 amino acids located in the endoplasmic reticulum (9). The biological function of ORMDL3 remains to be clarified, but a relationship between ORMDL3 and endoplasmic reticulum stress has been established. ORMDL3 was discovered by Moffatt et al (2) in 2007 and is the gene most definitively associated with pediatric asthma. The correlation between ORMDL3 and asthma has been confirmed in a number of ethnic populations (14), and increased expression of ORMDL3 is observed in more than one third of asthmatic children younger than 7 years of age (2). Epidemiology investigations related to the expression of ORMDL3 and pediatric asthma conducted in Beijing, Hong Kong and Chongqing in China have also suggested that ORMDL3 is closely associated with asthma in Han Chinese children (15-17).

The focus of research on ORMDL3 has turned from epidemiology to the mechanism of ORMDL3 in asthma. In this study, using an asthmatic airway remodeling model prepared by OVA sensitization and challenge, ORMDL 3 expression 
Table II. Pearson correlation analysis of Orosomucoid-like 3 with E-cad, FPS-1, VIM, Wat/Pbm and the percentage of collagen.

\begin{tabular}{lccccc}
\hline Analysis & E-cad & FSP-1 & VIM & Wat/Pbm & Collagen $(\%)$ \\
\hline Correlation index & -0.959 & 0.971 & 0.961 & 0.977 & 0.972 \\
P-value & $<0.0001$ & $<0.0001$ & $<0.0001$ & $<0.0001$ & $<0.0001$ \\
\hline
\end{tabular}

E-cad, E-cadherin; FSP-1, fibroblast-specific protein 1; VIM, vimentin; Wat, total bronchial wall area; Pbm, basal membrane perimeter.

A

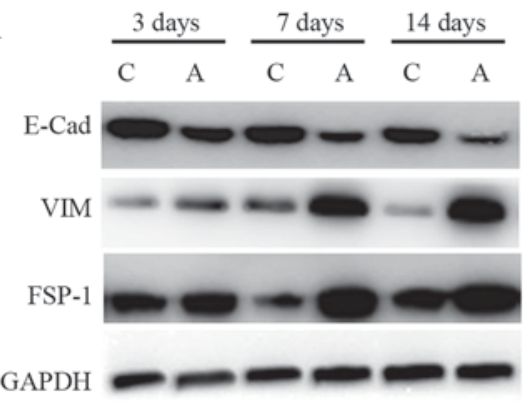

B

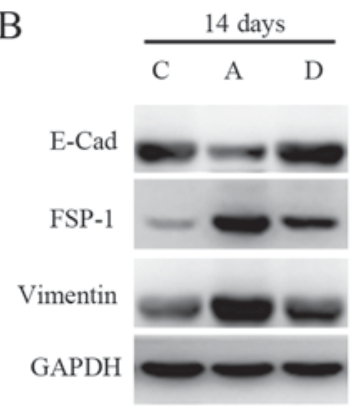

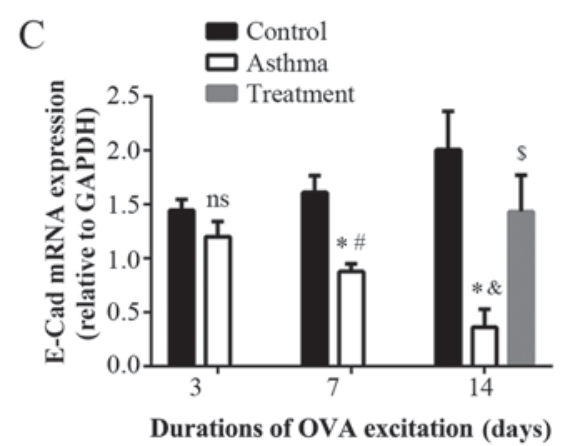

D

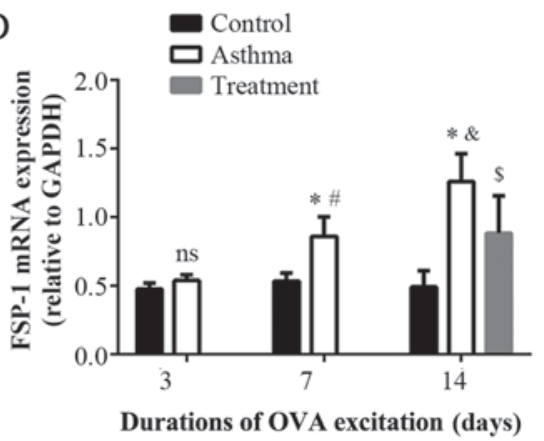

$\mathrm{E}$

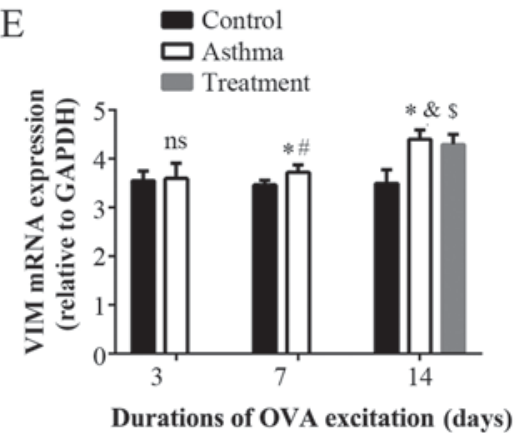

Figure 4. Protein and mRNA expression of epithelial-mesenchymal transition associated indicators induced by the development of asthma. (A) Western blotting of the relative protein expression of E-cad, VIM and FSP-1 in the lung tissues of the control and asthma groups at different time points. (B) Western blotting of the relative protein expression of E-cad, VIM and FSP-1 in the lung tissues of the control, asthma and treatment groups following 14 days exposure to OVA. Reverse transcription-quantitative polymerase chain reaction of the relative mRNA expression of (C) E-cad, (D) FSP-1 and (E) VIM in the lung tissues of the different groups. ${ }^{*} \mathrm{P}<0.01 \mathrm{vs}$. control; ${ }^{*} \mathrm{P}<0.01$ vs. asthma group at 3 days; ${ }^{\&} \mathrm{P}<0.01$ vs. asthma group at 7 days; ${ }^{\$} \mathrm{P}<0.01$ vs. treatment group. $\mathrm{C}$, control group; A, asthma group; D, dexamethasone treatment group; E-cad, E-cadherin; VIM, vimentin; FSP-1, fibroblast-specific protein 1; OVA, ovalbumin.

was observed in vivo during airway remodeling, and the EMT progression was detected in the bronchial epithelium. The relationship between ORMDL3 and EMT in bronchial epithelial cells was further confirmed in vitro.

Immunohistochemistry indicated that ORMDL3 was mainly expressed in bronchial epithelial cells in the asthma group but not in the control group, suggesting that ORMDL3 expression was associated with epithelial cell injury. Bronchial epithelial cells, which represent the front line of defense in the airway, are malleable. Following injury and during self-repair, some cells lose cell polarity and intercellular junctions, resulting in cytoskeletal remodeling, the emergence of interstitial cell phenotypes, and consequently, EMT. In the present study, the most severely injured portion of the lung tissue was the bronchial epithelium. Examination of the bronchial epithelium in the asthma group by light microscopy revealed cell edema, focal damage and detachment, while transmission electron microscopy indicated detachment of ciliated epithelial cells and disorganized cilia, increased cytoplasmic vacuoles, a significant increase in the secretory granules of goblet cell mucus, and slightly lax intracellular junctions. These observations indicated that significant damage and morphological changes occurred in bronchial epithelial cells in the asthma group.

In this research, changes in EMT-related indicators (E-cad, FSP-1, and VIM) were observed in lung tissues in the asthma group. FSP-1 belongs to the family of S100 calcium-binding proteins and is a typical marker of fibroblasts. It is mainly expressed at the transitional stage between epithelial cells and fibroblasts. FSP-1 is commonly used to detect the process of type II EMT because FSP-1 is present in most epithelial cells with type II EMT at the early stage of the process of organ fibrosis (18). E-cad is a marker of epithelial cells, and VIM is a marker of mesenchymal cells. Immunohistochemistry revealed increased FSP-1 in the bronchiolar epithelium in the asthma group, suggesting that some bronchiolar epithelial cells suffer EMT at an early stage. In addition, decreased E-cad expression and increased VIM expression were observed in the lung tissues of the asthma group, indicating early-stage fibrosis. In the asthma group, the expression of E-cad gradually declined 
A

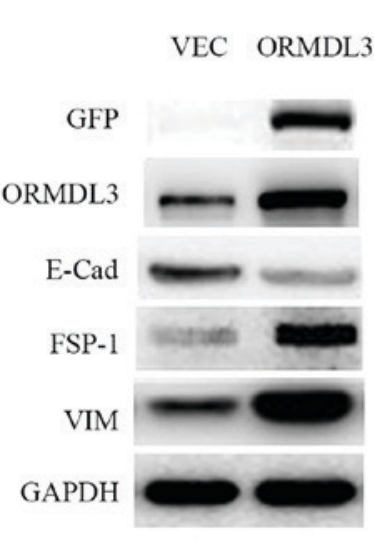

C

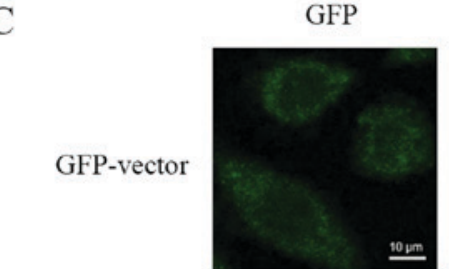

GFP-ORMDL3

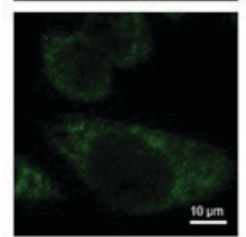

D

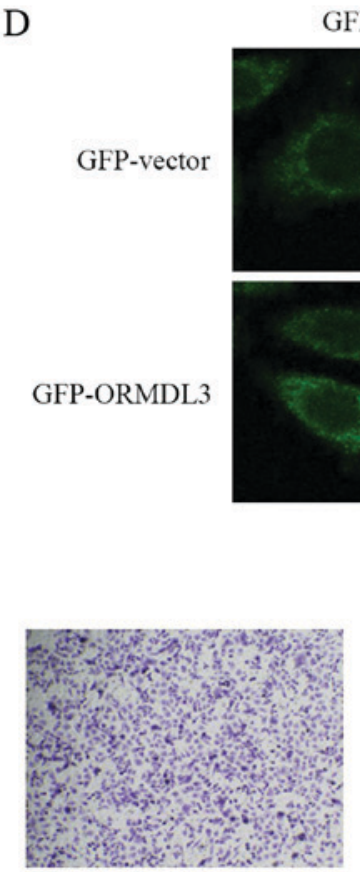

GFP-vector

GFP

E

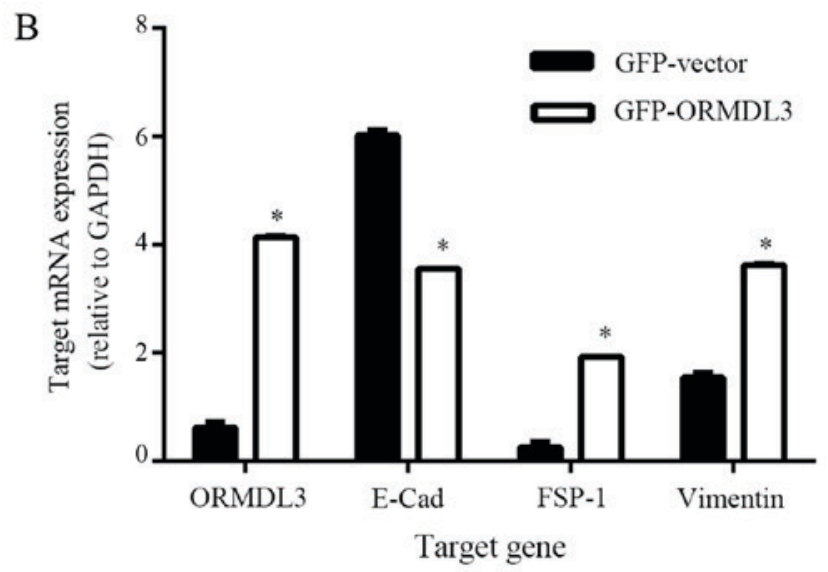

E-Cad
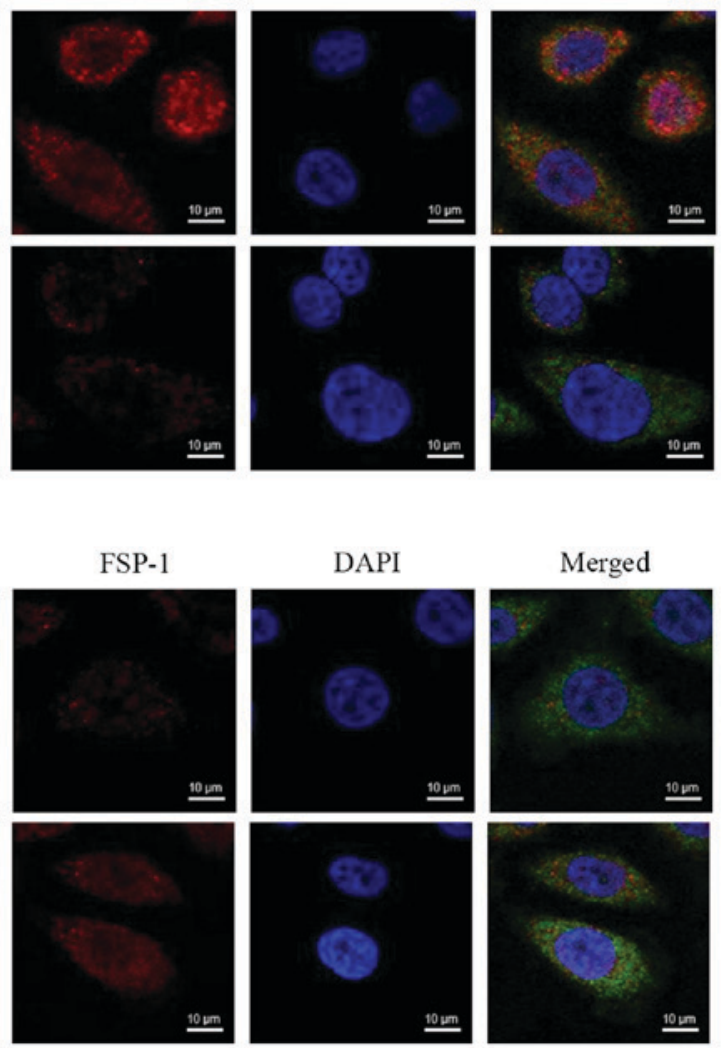

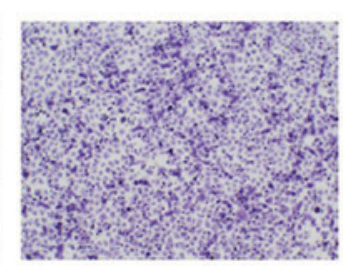

GFP-ORMDL3

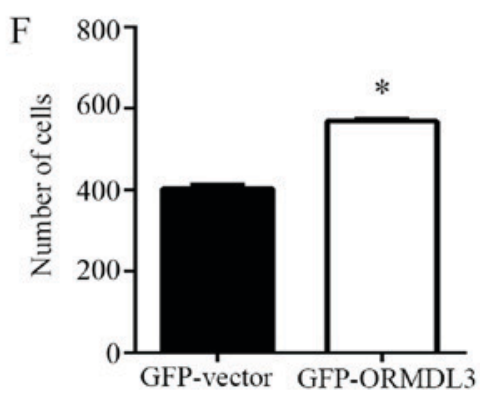

Figure 5. Transfection of ORMDL3 in the bronchial epithelial cell line 16HBE 14o- in vitro induced EMT. (A) The levels of the indicated proteins were determined by western blot analysis. Relative expression of the EMT indicators ORMDL3, E-cad, FSP-1 and VIM in 16HBE 14o- cells transfected with GFP-vector or GFP-ORMDL3 was assessed by western blotting and (B) reverse transcription-quantitative polymerase chain reaction. (C) Assessment of E-cad expression in 16 HBE 14o- cells transfected with GFP-vector or GFP-ORMDL3 by double-label confocal immunofluorescence microscopy (magnification, x600). (D) Assessment of FSP-1 expression in 16HBE 14o-cells transfected with GFP-vector or GFP-ORMDL3 by confocal immunofluorescence microscopy (magnification, x600). (E) Observation of the migration of 16HBE 14o-cells transfected with GFP-vector or GFP-ORMDL3 in a Transwell chamber using an inverted microscope (magnification, x100) and (F) determination of the number of 16HBE-14o cells in the two groups. "P<0.01 vs. GFP-vector. ORMDL3, orosomucoid-like 3; EMT, epithelial-mesenchymal transition; E-cad, E-cadherin; VIM, vimentin; FSP-1, fibroblast-specific protein 1; GFP, green fluorescent protein; VEC, GFP vector. 
and the expression of FSP-1 and VIM gradually increased in lung tissues with increasing duration of OVA challenge $(3,7$ and 14 days), indicating aggravation of EMT. Most importantly, the expression of ORMDL3 also increased with increasing duration of OVA challenge and was positively correlated with the degree of severity of airway remodeling and the expression of EMT-related indicators. When a plasmid expressing ORMDL3 was transfected into $16 \mathrm{HBE}-14 \mathrm{o}$ cells, E-cad expression decreased, FSP-1 and VIM expression increased, and cellular migration ability was enhanced, indicating induction of EMT. Therefore, we infer that ORMDL3 may be involved in the development of airway remodeling in asthma, consistent with the results of previous studies. Miller et al (7) have reported that airway remodeling changes similar to those observed in asthma, including bronchial smooth muscle thickening, increased fibers under basilar membranes and mucus in the lumen, appear in the lung tissues of ORMDL3 transgenic mice. Moreover, the present study reveals a dynamic role of ORMDL3 in the progression of asthma, as the level of ORMDL3 expression was correlated with the progression of asthmatic airway remodeling, and suggests that the overexpression of ORMDL3 may cause EMT. ORMDL3 might aggravate asthmatic airway remodeling by causing bronchial epithelial EMT, a novel aspect of the mechanism of ORMDL3 in asthma.

Yu et al (19) have also reported ORMDL3 was associated with airway remodeling in asthma via the ERK/MMP-9 pathway. Our study had some similarity to Yu et al (19) about the methods, the purpose and the results. Two papers both investigated the mechanism of ORMDL3 in airway remodeling in asthma. Yu et al (19) focuses on the study of the relationship between ORMDL3 and ERK/MMP-9 channels, while our paper focuses on the study of the relationship between ORMDL3 and EMT. But Yu et al (19) did not explain the direct relationship between the ORMDL3 and the p-ERK/MMP-9 pathway in vivo, nor did it indicate whether ORMDL3 regulates p-ERK/MMP-9 or p-ERK/MMP-9 regulates ORMDL3. Otherwise, it is known that Inhaled corticosteroids is the first choice to treat asthma. Studies has also been demonstrated that budesonide inhibits the p-ERK/MMP-9 pathway and ORMDL3 $(20,21)$. p-ERK/MMP-9 pathway is one of the classic airway remodeling pathways. Thus, budesonide treatment is more likely to directly inhibit p-ERK/MMP-9, leading to reduction in airway remodeling, rather than the reduced expression of ORMDL3 affect the p-ERK/MMP-9 pathway resulted in reduction in airway remodeling. There was lack of evidence to prove the relationship between ORMDL3, p-ERK/MMP-9 pathway and airway remodeling in Yu et al's paper (19). Airway remodeling in asthma includes smooth muscle cell proliferation and hypertrophy, extracellular matrix deposition, basement membrane thickening, inflammatory cell infiltration and gland proliferation, hypertrophy and epithelial-mesenchymal transition. This study focuses on the effect of ORMDL3 on airway remodeling in asthma by EMT. This paper shows the airway remodeling in asthma in different periods, the expression of ORMDL3 was gradually increased with EMT process including airway wall thickness and collagen deposition, and the epithelial cell marker (E-cad negative correlation, mesenchymal cell markers FSP-1 and Vimentin positive correlation). In addition, cell experiments in vitro have shown that overexpression of ORMDL3 could induce epithelial EMT. Thus, the relationship between ORMDL3 and airway remodeling and EMT was clearly demonstrated. The similarity of the detection methods (H\&E-staining, Masson-staining and immunohistochemistry), and both of the two specimens were lung tissues of $\mathrm{BALB} / \mathrm{c}$ mice, thus, the pictures of the two articles were similar. But carefully distinguish, there was difference between the two papers. First of all, the image of color and the shades of staining are different. Secondly, subjects of Yu et al (19) were divided into three groups (asthma group, budesonide treatment group, control group). In our paper, subjects were also divided into 3 groups too (control group, asthma group and treatment group), and according to the different time of allergen challenge $3 \mathrm{rd}$, 7th, 14th, control group and asthma group divided into 6 groups (control group for 3 days, control group for 7 days, control group for 14 days, and asthma group for 3 days, asthma group for 7 days, asthma group for 14 days). Finally, our purpose was to observe the relationship of ORMDL3 expression and morphological changes in different period of airway remodling. And the research content and results displayed in our pictures are different from Yu et al (19).

The process of EMT is regulated by a variety of cytokines and signaling pathways, but the signaling pathway by which ORMDL3 affects bronchiolar EMT is unknown. Two major mechanisms of ORMDL3 have been revealed in previous studies: 1) ORMDL3 co-localizes with sarcoplasmic reticulum Ca-ATPase on endoplasmic reticulum membranes (SERCA) to inhibit SERCA function, increase calcium ions in the cytoplasm and reduce calcium irons in the endoplasmic reticulum, leading to endoplasmic reticulum calcium imbalance, subsequent endoplasmic reticulum stress, and the unfolded protein response (22). 2) ORMDL3 combines with serine palmitoyltransferase to form the SPOTS complex and decrease the enzymatic activity of serine palmitoyltransferase. As this enzyme catalyzes the rate-limiting step in sphingolipid production, formation of the complex with ORMDL3 could negatively regulate sphingolipid production (23). EMT occurs when lung epithelial cells are subjected to the endoplasmic reticulum stress response (24), whereas abnormal sphingolipids cause mast cell degranulation, bronchial hyperreactivity and the migration of immune cells (25). However, whether ORMDL3 causes bronchiolar epithelial EMT changes via the endoplasmic reticulum stress reaction and inhibition of sphingomyelin production remains to be further verified.

In summary, this study has revealed that ORMDL3 is associated closely with asthmatic airway remodeling and the progression of bronchiolar EMT and may cause bronchial epithelial EMT. A further understanding of the role of the dynamic changes in ORMDL3 in the development of asthma may guide clinical applications. ORMDL3 may be useful as an index to monitor asthmatic airway remodeling or its severity and is likely to be a new precision medicine therapeutic target for asthma, especially for refractory cases.

\section{Acknowledgements}

We would like to acknowledge the 'Clinical Ability Construction Projects of Liaoning Province' (serial no. LNCCC-C02-2015) for the financial support of this study. 


\section{References}

1. Anderson GP: Endotyping asthma: New insights into key pathogenic mechanisms in a complex, heterogeneous disease. Lancet 372: 1107-1119, 2008.

2. Moffatt MF, Kabesch M, Liang L, Dixon AL, Strachan D, Heath S, Depner M, von Berg A, Bufe A, Rietschel E, et al: Genetic variants regulating ORMDL3 expression contribute to the risk of childhood asthma. Nature 448: 470-473, 2007.

3. Balantic M, Rijavec M, Flezar M, Camlek T, Hudoklin I, Kosnik M, Korosec P and Suskovic S: A polymorphism in ORMDL3 is associated not only with asthma without rhinitis but also with chronic obstructive pulmonary disease. J Investig Allergol Clin Immunol 23: 256-261, 2013.

4. Holgate ST: The sentinel role of the airway epithelium in asthma pathogenesis. Immunol Rev 242: 205-219, 2011.

5. Hackett TL: Epithelial-mesenchymal transition in the pathophysiology of airway remodelling in asthma. Curr Opin Allergy Clin Immunol 12: 53-59, 2012.

6. Johnson JR, Roos A, Berg T, Nord M and Fuxe J: Chronic respiratory aeroallergen exposure in mice induces epithelial-mesenchymal transition in the large airways. PLoS One 6: e16175, 2011

7. Miller M, Rosenthal P, Beppu A, Mueller JL, Hoffman HM, Tam AB, Doherty TA, McGeough MD, Pena CA, Suzukawa M, et al: ORMDL3 transgenic mice have increased airway remodeling and airway responsiveness characteristic of asthma. J Immunol 192: 3475-3487, 2014.

8. Miller M, Tam AB, Cho JY, Doherty TA, Pham A, Khorram N, Rosenthal P, Mueller JL, Hoffman HM, Suzukawa M, et al: ORMDL 3 is an inducible lung epithelial gene regulating metalloproteases, chemokines, OAS, and ATF6. Proc Natl Acad Sci USA 109: 16648-16653, 2012.

9. Hjelmqvist L, Tuson M, Marfany G, Herrero E, Balcells S and Gonzàlez-Duarte R: ORMDL proteins are a conserved new family of endoplasmic reticulum membrane proteins. Genome Biol 3: RESEARCH0027, 2002.

10. Meyer-Martin H, Reuter S and Taube C: Mouse models of allergic airway disease. Methods Mol Biol 1193: 127-141, 2014.

11. Shin YS, Takeda K and Gelfand EW: Understanding asthma using animal models. Allergy Asthma Immunol Res 1: 10-18, 2009.

12. James AL, Hogg JC, Dunn LA and Paré PD: The use of the internal perimeter to compare airway size and to calculate smooth muscle shortening. Am Rev Respir Dis 138: 136-139, 1988.

13. Palmans E, Kips JC and Pauwels RA: Prolonged allergen exposure induces structural airway changes in sensitized rats. Am J Respir Crit Care Med 161: 627-635, 2000.

14. Galanter J, Choudhry S, Eng C, Nazario S, Rodríguez-Santana JR, Casal J, Torres-Palacios A, Salas J, Chapela R, Watson HG, et al: ORMDL3 gene is associated with asthma in three ethnically diverse populations. Am J Respir Crit Care Med 177: 1194-1200, 2008 .
15. Leung TF, Sy HY, Ng MC, Chan IH, Wong GW, Tang NL, Waye MM and Lam CW: Asthma and atopy are associated with chromosome 17q21 markers in Chinese children. Allergy 64: 621-628, 2009.

16. Yu X, Yu C, Ren Z, Deng Y, Song J, Zhang H and Zhou H: Genetic variants of 17q21 are associated with childhood-onset asthma and related phenotypes in a northeastern Han Chinese population: A case-control study. Tissue Antigens 83: 330-336, 2014.

17. Zhe J, Xin C, Qiang W and Hong L: Study of Association between ORMDL3 expression level, life-style characters, indoor air qualifies and childhood asthma in Beijing. J Med Res 39: 22-25, 2010.

18. Donato R, Cannon BR, Sorci G, Riuzzi F, Hsu K, Weber DJ and Geczy CL: Functions of S100 proteins. Curr Mol Med 13: 24-57, 2013.

19. Yu F, Sun Y, Yu J, Ding Z, Wang J, Zhang L, Zhang T, Bai Y and Wang Y: ORMDL3 is associated with airway remodeling in asthma via the ERK/MMP-9 pathway. Mol Med Rep 15: 2969-2976, 2017

20. Rae MT, Price D, Harlow CR, Critchley HO and Hillier SG: Glucocorticoid receptor-mediated regulation of MMP9 gene expression in human ovarian surface epithelial cells. Fertil Steril 92: 703-708, 2009.

21. Zou LP,Zhang X,Zhang Y,Xu XJ and Wang TF: Down-regulatory effects of budesonide on expression of STAT6 and ORMDL3 in lung tissues of asthmatic mice. Zhongguo Dang Dai Er Ke Za Zhi 16: 198-202, 2014 (In Chinese).

22. Cantero-Recasens G, Fandos C, Rubio-Moscardo F, Valverde MA and Vicente R: The asthma-associated ORMDL3 gene product regulates endoplasmic reticulum-mediated calcium signaling and cellular stress. Hum Mol Genet 19: 111-121, 2010.

23. Siow D, Sunkara M, Dunn TM, Morris AJ and Wattenberg B: ORMDL/serine palmitoyltransferase stoichiometry determines effects of ORMDL3 expression on sphingolipid biosynthesis. J Lipid Res 56: 898-908, 2015.

24. Tanjore H, Cheng DS, Degryse AL, Zoz DF, Abdolrasulnia R, Lawson WE and Blackwell TS: Alveolar epithelial cells undergo epithelial-to-mesenchymal transition in response to endoplasmic reticulum stress. J Biol Chem 286: 30972-30980, 2011.

25. Breslow DK, Collins SR, Bodenmiller B, Aebersold R, Simons K, Shevchenko A, Ejsing CS and Weissman JS: Orm family proteins mediate sphingolipid homeostasis. Nature 463: 1048-1053, 2010 